\title{
Game-Theoretic Approach to Capacity and Stability Evaluations of Decentralized Adaptive Route Selections in Wireless Ad Hoc Networks
}

\author{
Koji YAMAMOTO $^{\dagger a)}$, Student Member and Susumu YOSHIDA ${ }^{\dagger}$, Fellow
}

\begin{abstract}
SUMMARY A game-theoretic analysis is applied to the evaluation of capacity and stability of a wireless ad hoc network in which each source node independently chooses a route to the destination node so as to enhance throughput. First, the throughput of individual multihop transmission with rate adaptation is evaluated. Observations from this evaluation indicate that the optimal number of hops in terms of the achievable end-to-end throughput depends on the received signal-to-noise ratio. Next, the decentralized adaptive route selection problem in which each source node competes for resources over arbitrary topologies is defined as a game. Numerical results reveal that in some cases this game has no Nash equilibria; i.e., each rational source node cannot determine a unique route. The occurrence of such cases depends on both the transmit power and spatial arrangement of the nodes. Then, the obtained network throughput under the equilibrium conditions is compared to the capacity under centralized scheduling. $\mathrm{Nu}-$ merical results reveal that when the transmit power is low, decentralized adaptive route selection may attain throughput near the capacity.

key words: ad hoc networks, decentralized control, game theory, network capacity, routing
\end{abstract}

\section{Introduction}

Recent advancements in radio technologies and the success of the Internet have shown the potential of information systems as ubiquitous networks accessible from anywhere, at any time, and with any device [1]. A key enabling technology for such networks is wireless ad hoc networking.

Wireless ad hoc networks are self-configuring systems formed by co-operating nodes without any established infrastructure or centralized administration. A key assumption is that any two nodes can communicate directly if there is an adequate radio propagation path between them subject to the maximum transmit power constraints of the nodes. Packets are relayed from the source to the final destination, which can be relatively far apart, by multihop transmission between pairs of nodes [2].

The characteristics of ad hoc networks, including possible node mobility, lack of centralized control, constraints on energy consumption, and bandwidth-constrained wireless links, have led to the creation of widespread research fields [3]. Research in the area of ad hoc networks has been concentrated on the network layer [4]-[6], the medium access control (MAC) layer, and the physical layer. Crosslayer design that takes into account the characteristics of the

\footnotetext{
Manuscript received July 23, 2004.

${ }^{\dagger}$ The authors are with the Graduate School of Informatics, Kyoto University, Kyoto-shi, 606-8501 Japan.

a)E-mail: kyamamot@hanase.kuee.kyoto-u.ac.jp DOI: 10.1093/ietcom/e88-b.3.1009
}

underlying layers can provide optimizations across multiple layers [7], [8].

The capacity of ad hoc networks has attracted much interest in recent years [9]-[12]. Gupta et al. estimated the throughput per source-destination pair in a large-scale ad hoc network with optimal routing and scheduling [9]. Toumpis et al. defined "capacity region" as the achievable capacity of a wireless ad hoc network and evaluated various transmit schemes including routing, scheduling, and power control [11]. Optimal routing and scheduling require coordination among all the nodes in the network.

The decentralization and distribution of control in ad hoc networks mean that such networks are inherently scalable. However, the natural conflict among nodes in a network due to selfishness cannot always attain throughput near the capacity. A promising approach to analyzing decentralized control and node selfishness is game theory, which deals with how individuals interact and compete for resources. The effect of "selfish" random access has been evaluated using game-theoretic analysis [13], [14]; an algorithm has been proposed for deciding whether to accept or reject a relay request for multihop transmission [15]; and studies have been made of decentralized power control problems in which each node chooses its power level for establishing connectivity between the source and destination [16].

This paper reports a game-theoretic analysis of a decentralized adaptive route selection (DARS) problem in a wireless ad hoc network, in which multiple decision-making nodes select a route to the destination so as to enhance their own throughput. This problem is similar to that defined by Eidenbenz et al. [16]. However, the focus here is not connectivity but the capacity of bandwidth-constrained wireless networks.

The remainder of this paper is organized as follows: In Sect. 2, we discuss a system model and present throughput analysis of individual multihop transmission with rate adaptation. In Sect. 3, we introduce the normal form game and define the DARS game. In Sect. 4, we investigate the Nash equilibria of the DARS game. We also analyze the achievable throughput of a network under DARS and compare it to the capacity region [11]. Sect. 5 concludes the paper with a summary and some final remarks about the limited scope of this paper. 
Table 1 Parameters used in the evaluation.

\begin{tabular}{c|c}
\hline Parameters & Values \\
\hline System area & $20 \mathrm{~m} \times 20 \mathrm{~m}$ \\
Bandwidth $W$ & $1 \mathrm{MHz}$ \\
Noise power spectral density $\eta$ & $10^{-15} \mathrm{~W} / \mathrm{Hz}$ \\
Carrier frequency & $2 \mathrm{GHz}$ \\
Antenna gain & $0 \mathrm{dBi}$ \\
Path loss & Free space \\
\hline
\end{tabular}

\section{Wireless Ad Hoc Network Model}

Table 1 summarizes the parameters we used in our evaluation. $N$ nodes are uniformly and independently distributed in a two-dimensional square area and are stationary throughout the evaluation. We assume that each node can be either transmitting or receiving at a given time and can serve as a repeater for only one other source node. Free space path loss is assumed between any two nodes. The effects of shadowing and fast fading are not considered for the sake of simplicity, as has been done in [7]. Omnidirectional antennas are used for the nodes. Under these assumptions, the power gain between nodes $i$ and $j$ is given by

$$
G_{i j}=\left(\frac{\lambda}{4 \pi d_{i j}}\right)^{2},
$$

where $\lambda$ represents the wavelength and $d_{i j}$ represents the distance between nodes $i$ and $j$.

\subsection{Rate Adaptation and Resulting Physical Layer Bit Rate}

Let $P_{i}$ denote the transmit power of node $i$, so that node $j$ receives a signal with power $G_{i j} P_{i}$. Let $\mathcal{T}$ be the subset of nodes simultaneously transmitting at any given time. When node $j(\notin \mathcal{T})$ is receiving a signal from node $i(\in \mathcal{T})$, the received signal-to-interference plus noise ratio (SINR) at node $j$ is

$$
\gamma_{i j}=\frac{G_{i j} P_{i}}{\eta_{j} W+\sum_{k \in \mathcal{T}, k \neq i} G_{k j} P_{k}},
$$

where $\eta_{j}$ represents the noise power spectral density at receiving node $j$ and $W$ represents the bandwidth. All interference signals are assumed to be equivalent to additive white Gaussian noise over the signal bandwidth.

We assume that transmitting node $i$ adjusts transmission rate $r_{i j}$ based on the received SINR $\gamma_{i j}$, and that the signal can be received with a negligible probability of error at receiving node $j$. Shannon capacity is used as the specific dependence of $r_{i j}$ on $\gamma_{i j}$ as used in [11]:

$$
r_{i j}=W \log _{2}\left(1+\gamma_{i j}\right)
$$

\subsection{Time and Packet Scheduling}

The achievable throughput at the MAC layer in wireless networks with only single-hop transmission depends on the physical layer bit rate, the efficiency of the MAC layer, the size of the packets, and the number of nodes in the network [17], [18]. Since the main purpose of this paper is to investigate the performance of the DARS game, no coordinated time and packet scheduling among nodes is assumed.

In order to evaluate the throughput of wireless networks with multihop transmission, we use the average endto-end throughput assuming that the data size of every packet is equal and that as soon as the destination node receives a packet, the source node transmits the next packet. We refer to this throughput as the capacity of the network for the given packet scheduling.

\subsection{End-to-End Throughput of Individual Multihop Trans- mission with Rate Adaptation}

Consider end-to-end throughput of individual multihop transmission assuming that there is no interference. Under this assumption, the SINR reduces to the signal-to-noise ratio (SNR). The transmit power of every node is assumed to be equal to $P$. Let $\eta$ denote the power spectral density of the thermal noise. Using Eqs. (1), (2), and (3), we get end-toend throughput of single-hop transmission of

$$
C_{1}=W \log _{2}\left[1+\frac{P}{\eta W}\left(\frac{\lambda}{4 \pi d}\right)^{2}\right],
$$

where $d$ represents the distance between the source and destination nodes.

We model the topology of $n$-hop transmission as equally spaced $(n-1)$ relaying nodes on a straight line segment from the source node to the destination node and assume that spatial reuse is not allowed. While these assumptions are not necessarily realistic, they do enable us to gain insight into the design of wireless ad hoc networks. We can switch from single-hop transmission to $n$-hop transmission under these assumptions by allowing only one node at a time to transmit. The percentage of time a node can transmit a signal becomes approximately $1 / n$, and the per hop distance is reduced to $d / n$. Consequently, the end-to-end throughput of $n$-hop transmission under these assumptions is

$$
C_{n}=\frac{W}{n} \log _{2}\left[1+\frac{P}{\eta W}\left(\frac{\lambda}{4 \pi d / n}\right)^{2}\right] .
$$

Figure 1 shows the distance dependence of the end-toend throughput of $n$-hop transmission $(n=1,2,3,10)$. For a long enough distance, multihop transmission may result in maximum throughput. Equation (5) suggests that the endto-end throughput also depends on the transmit power of each node (which is discussed in more detail in [12]). Therefore, multihop transmission may be more effective under low SNR conditions. Figure 2 shows that the optimal number of hops in terms of the achievable end-to-end throughput depends on the SNR of the received signal. 


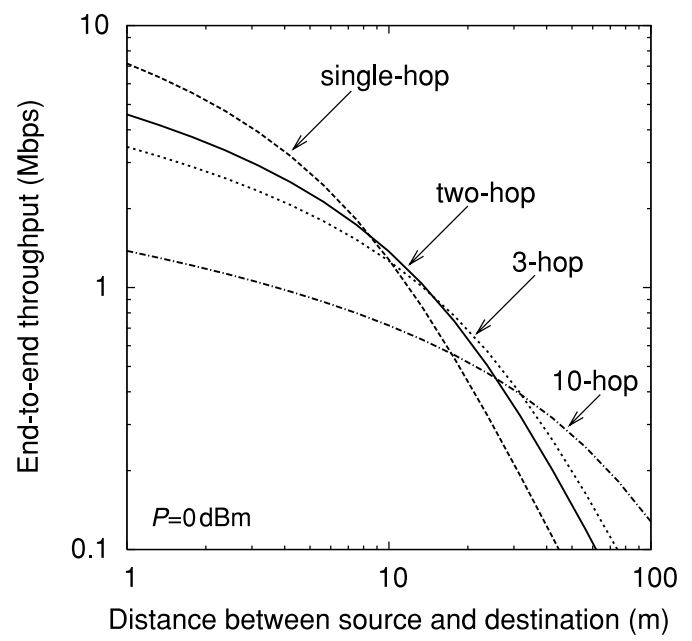

Fig. 1 Dependence of end-to-end throughput of multihop transmission on distance between source and destination nodes.

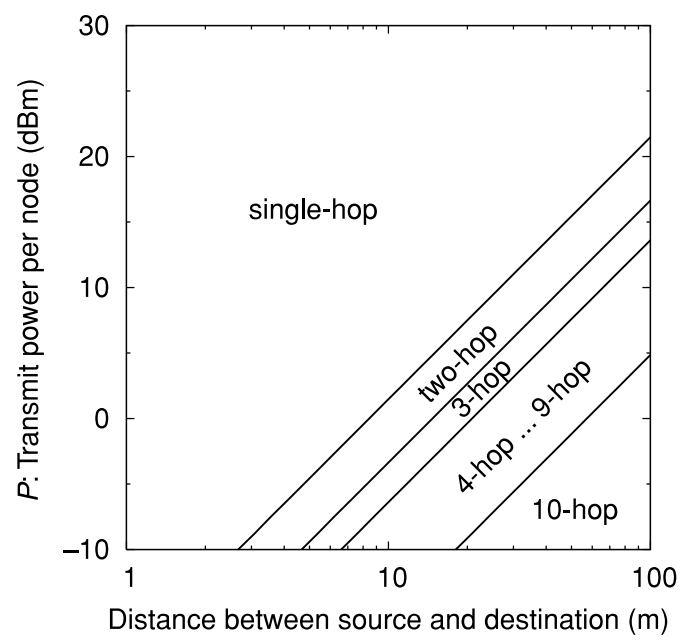

Fig. 2 Optimal number of hops in terms of achievable end-to-end throughput.

\section{Game-Theoretic Framework}

\subsection{Normal Form Game}

A game in normal form has three aspects. First, there is a set of players, $\mathcal{I}$, which we take to be the finite set $\{1,2, \ldots, I\}$. Second, there is a set of pure strategies, $S_{i}(i \in \mathcal{I})$, available to each player. Finally, there is a set of payoff functions, $u_{i}$. Each player has a preference for an outcome. The payoff function represents the player's preference and maps the player's anticipated outcome given his or her selected strategy to a real number. In the game, each player's objective is to select a strategy, $s_{i} \in S_{i}$, so as to maximize his or her own payoff function. A mixed strategy for player $i$ is a probability distribution over pure strategies $S_{i}$. In this paper, unless otherwise noted, we assume pure strategies since mixed strategies do not seem reasonable in studies of stability (similar arguments are presented in [16], [19]).
A fundamental solution concept in game theory is a Nash equilibrium, which is a point where neither player gains by changing his or her strategy unilaterally, so that neither player has an incentive to deviate [20]. A pure strategy profile, $\boldsymbol{s}^{*}=\left(s_{1}{ }^{*}, \ldots, s_{I}{ }^{*}\right)$, is a Nash equilibrium if, for all players $i$,

$$
\begin{array}{r}
u_{i}\left(s^{*}\right) \geq u_{i}\left(s_{1}{ }^{*}, \ldots, s_{i-1}{ }^{*}, s_{i}, s_{i+1}{ }^{*}, \ldots, s_{I}{ }^{*}\right) \\
\forall s_{i} \in S_{i} .
\end{array}
$$

In particular, for a two-player game, a pure strategy profile is a Nash equilibrium if

$$
\begin{array}{ll}
u_{1}\left(s_{1}{ }^{*}, s_{2}{ }^{*}\right) \geq u_{1}\left(s_{1}, s_{2}{ }^{*}\right) & \forall s_{1} \in S_{1} \\
u_{2}\left(s_{1}{ }^{*}, s_{2}{ }^{*}\right) \geq u_{2}\left(s_{1}{ }^{*}, s_{2}\right) & \forall s_{2} \in S_{2} .
\end{array}
$$

\subsection{Decentralized Adaptive Route Selection Game}

Let us assume source nodes are players in a game. We define a DARS game in which each source node has to select a route to the destination node so that the maximum end-toend throughput is achieved. Therefore, the set of all possible single-hop and multihop routes is a set of strategies.

We assume that the source nodes have perfect knowledge of the power gain between each transmitting and receiving node pair, the transmit power of each transmitting node, and the noise power spectral density at each receiving node. This assumption is necessary for comparing the achievable throughput under decentralized scheduling and the capacity under centralized scheduling with perfect knowledge. Under this assumption, each source node can estimate the end-to-end throughput of all source nodes as a consequence of their route selections.

\section{Behavior of DARS Game and Resulting Throughput}

In this section, we will demonstrate that it is useful to classify the network topologies based on the number of pure strategy Nash equilibria. The first three subsections describe how the DARS game behaves in a network topology with a unique equilibrium, without equilibria, and with multiple equilibria. We estimate network throughput under equilibrium conditions (hereinafter referred to as "equilibrium throughput"). This equilibrium throughput is a reasonable payoff as a result of the DARS game. We then compare the equilibrium throughput and capacity under the given packet scheduling.

For the sake of simplicity, we consider the case where there are only two source-destination pairs and the number of hops is limited to two. This is a reasonable assumption because the purpose of this paper is to evaluate the impact of the DARS game among multiple decision-making entities in wireless ad hoc networks. Under this assumption, Nash equilibria can be found using Eq. (7). Since we are interested in the maximum throughput, the control overhead associated with route selection is not taken into account. 


\subsection{Network Topology with a Unique Equilibrium}

Figure 3 shows an example network in which the DARS game has a unique equilibrium. In this network, there are two communication pairs: source node 1 to destination node 2 and source node 3 to destination node 4 . The number of equilibria depends on the transmit power of the nodes as well as on their spatial arrangements. We will discuss the transmit power dependence of the number of equilibria later in this section.

For given source node $N_{S}$ and destination node $N_{D}$, let $\left(N_{S} \rightarrow N_{R} \rightarrow N_{D}\right)$ represent multihop transmission using node $N_{R}$ as a relaying node. Figure 3 shows that source node 1 's equilibrium route is $(1 \rightarrow 13 \rightarrow 2)$ and source node 3 's equilibrium route is $(3 \rightarrow 14 \rightarrow 4)$.

Every point in Fig. 4 represents a possible combination of the throughput of source node 1 and that of source node 3. Particularly, each point on the axes corresponds to

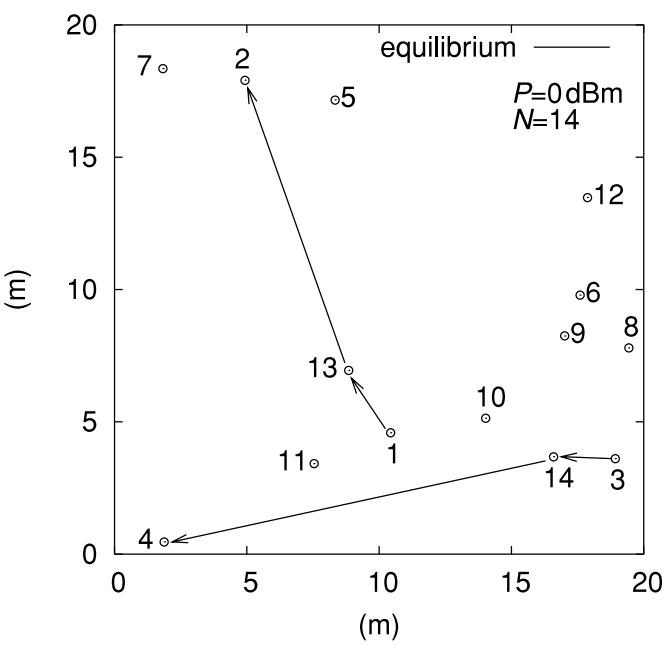

Fig.3 Example network in which DARS game has a unique equilibrium.

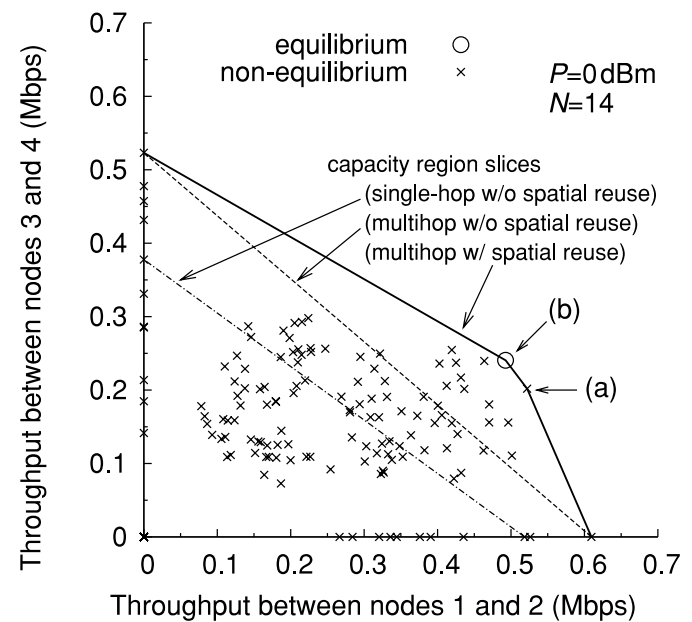

Fig. 4 Capacity region and equilibrium throughput of network shown in Fig. 3. the throughput of individual source-destination pairs. Each curve represents a two-dimensional slice of the capacity region [11] for the following three particular strategies.

- Single-hop transmission without spatial reuse

- Multihop transmission without spatial reuse

- Multihop transmission with spatial reuse

The capacity region is a multidimensional region that contains all achievable combinations of throughput between the nodes in the network. If centralized time scheduling can be used, changing the time devoted to the respective route profiles enables different points on the line segment defined by two arbitrary points to be achieved. These different points compose the capacity region (explained in more detail in [11]). A slice through the capacity region under the assumption of single-hop transmission without spatial reuse is a straight line. With the introduction of multihop transmission and spatial reuse, the size of the capacity region increases. Hereafter, the capacity region slice under multihop transmission with spatial reuse is treated.

Figure 4 indicates that two points (a) and (b) are on the capacity region slice except for the points on the axes. These two points correspond to the following route profiles.

(a) $(1 \rightarrow 13 \rightarrow 2),(3 \rightarrow 4)$

(b) $(1 \rightarrow 13 \rightarrow 2)$, $(3 \rightarrow 14 \rightarrow 4)$

Route profile (a), in which source node 3 selects single-hop transmission, is not an equilibrium. Route profile (b) is an equilibrium because source node 1 selects $(1 \rightarrow 13 \rightarrow 2)$ in both profiles, so the selection of these two route profiles depends only on the preference of source node 3 .

\subsection{Network Topology with No Equilibria}

The DARS game does not always have Nash equilibria in all networks. Figure 5 shows an example network in which the DARS game has no equilibria. In this case, for all strategy pairs, at least one source node has an incentive to deviate. Consider the following four route profiles.

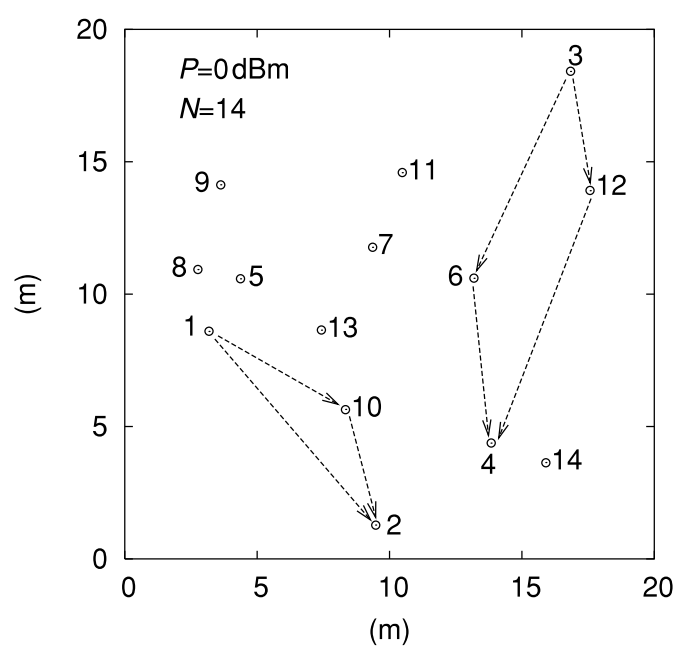

Fig.5 Example network in which DARS game has no equilibria. 


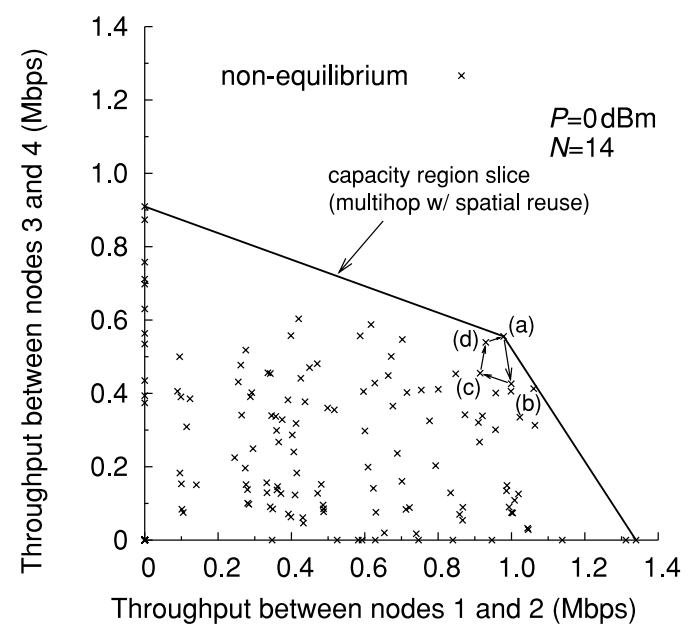

Fig. 6 Capacity region and equilibrium throughput of network shown in Fig. 5.

(a) $(1 \rightarrow 2),(3 \rightarrow 12 \rightarrow 4)$

(b) $(1 \rightarrow 10 \rightarrow 2),(3 \rightarrow 12 \rightarrow 4)$

(c) $(1 \rightarrow 10 \rightarrow 2),(3 \rightarrow 6 \rightarrow 4)$

(d) $(1 \rightarrow 2),(3 \rightarrow 6 \rightarrow 4)$

Figure 6 shows the capacity region slice and the combination of throughput for each strategy pair. The four points connected with arrows correspond to the four route profiles above.

The non-existence of equilibria has the following technical aspect. If the DARS game starts at point (a), source node 1 switches to $(1 \rightarrow 10 \rightarrow 2)$, resulting in point $(b)$, and then source node 3 switches to $(3 \rightarrow 6 \rightarrow 4)$, resulting in point (c). The cycle (d), (a), (b), (c) will simply repeat and never converge. Therefore, the non-existence of pure strategy Nash equilibria can be used for analyzing the instability of the route selection.

Consequently, in some cases, even with perfect information except for knowledge of the other nodes' decisions, each rational node cannot determine a unique route. In these cases, the best strategy is determined based on a probability distribution.

\subsection{Network Topology with Multiple Equilibria}

In some networks, the DARS game has multiple Nash equilibria, as shown by the example in Fig. 7. In this network, the following two route profiles are both Nash equilibria.

(a) $(1 \rightarrow 8 \rightarrow 2),(3 \rightarrow 14 \rightarrow 4)$

(b) (1 $\rightarrow 14 \rightarrow 2)$, (3 $\rightarrow 4)$

Source nodes 1 and 3 both try to use the same node, 14 .

Figure 8 , which shows the capacity region slice and the combination of throughput for each strategy pair, reveals that these equilibria are not Pareto optimal. A Pareto optimal outcome is one that cannot be improved without reducing the payoff of at least one player. This is because if a route profile on the capacity region is selected, at least one source node has an incentive to deviate. Therefore, the other source

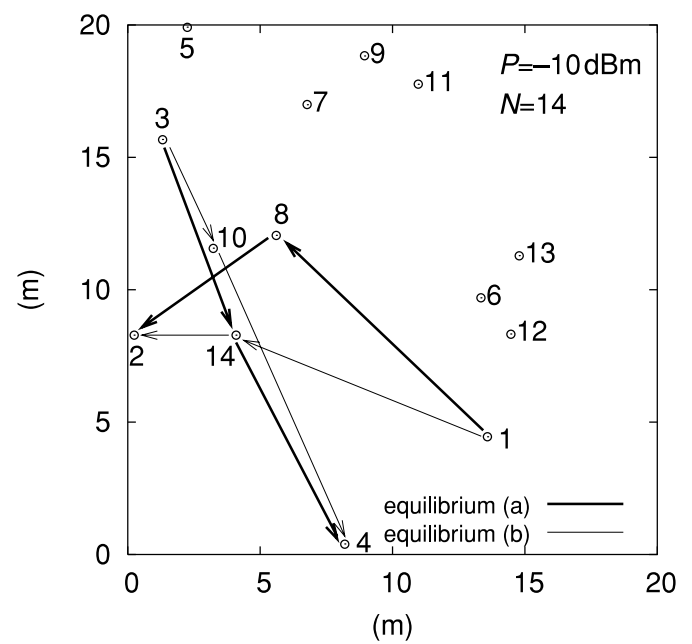

Fig. 7 Example network in which DARS game has multiple equilibria.

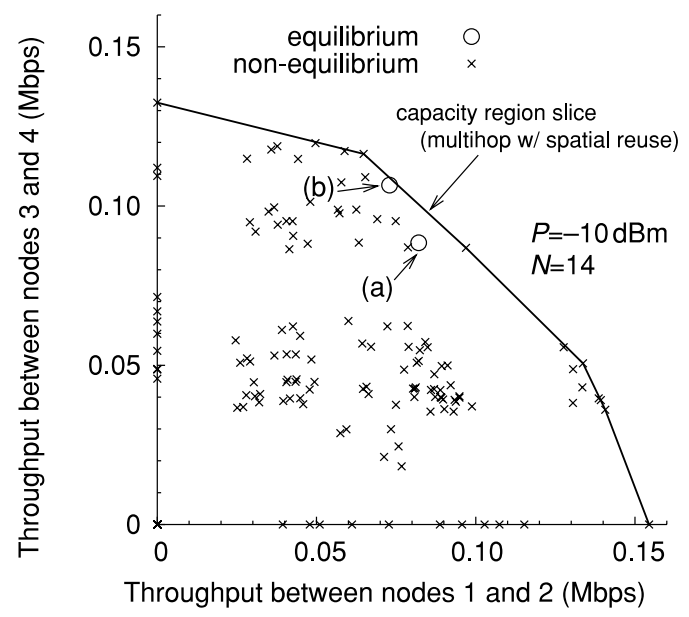

Fig. 8 Capacity region and equilibrium throughput of network shown in Fig. 7.

node does not select strategies corresponding to this route profile. To reach capacity in these cases, centralized control may be required.

\subsection{Average Capacity}

As mentioned above, the behavior of the DARS game varies depending on the spatial arrangements of the nodes and can be classified based on the number of equilibria: none, a unique equilibrium, and multiple equilibria. In this subsection, to evaluate the performance of the DARS game for various node arrangements, we classify the many possible node arrangements into these three categories.

Figure 9 shows the percentage of node arrangements in each category as a function of the transmit power per node. The DARS game has a unique equilibrium in $90 \%$ or more of the arrangements. The percentage of networks in which there are no Nash equilibria increases with the transmit power per node. This means that with low power levels, the interference between two source-destination pairs 


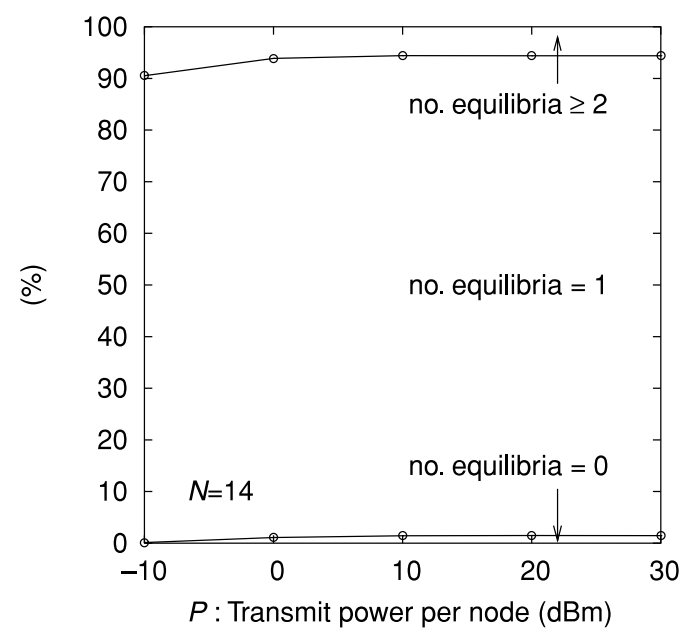

Fig. 9 Percentage of three categories based on number of equilibria as function of transmit power per node.

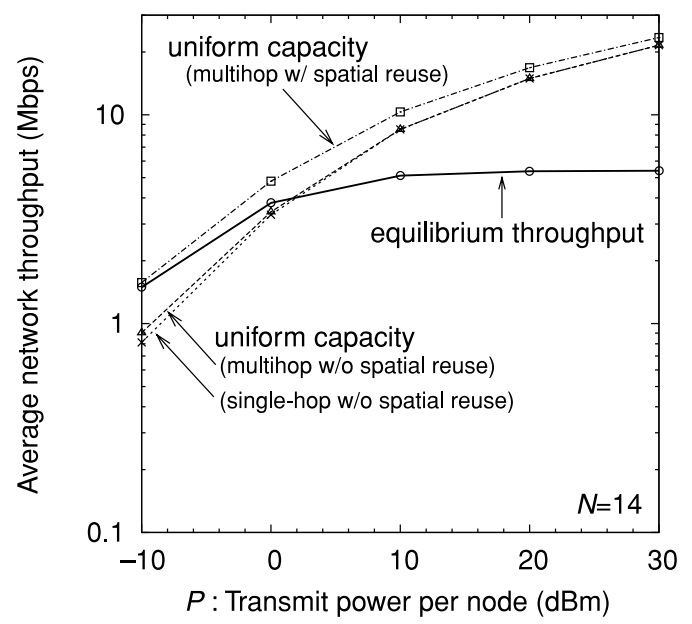

Fig. 10 Power dependence of average network throughput.

is negligible, so route selection can be determined without considering other source nodes.

Figure 10 shows the transmit power dependence of the average network throughput for the networks with at least one Nash equilibrium. The dashed and dotted lines represent the uniform capacity, which is defined as the total capacity under the assumption that all source nodes have the same end-to-end throughput. It thus corresponds to the boundary of the capacity region (explained in detail in [7], [11]). The introduction of multihop transmission increases the uniform capacity under the condition that the transmit power is low. This result can be explained by the finding that multihop transmission is effective under low SNR conditions, as presented in Sect. 2.3.

The introduction of spatial reuse in addition to multihop transmission increases the uniform capacity, especially when the transmit power is low. This is because the capacity of wireless ad hoc networks with spatial reuse is constrained by the interference of concurrent transmissions.

The solid line in Fig. 10 represents the average equilib-

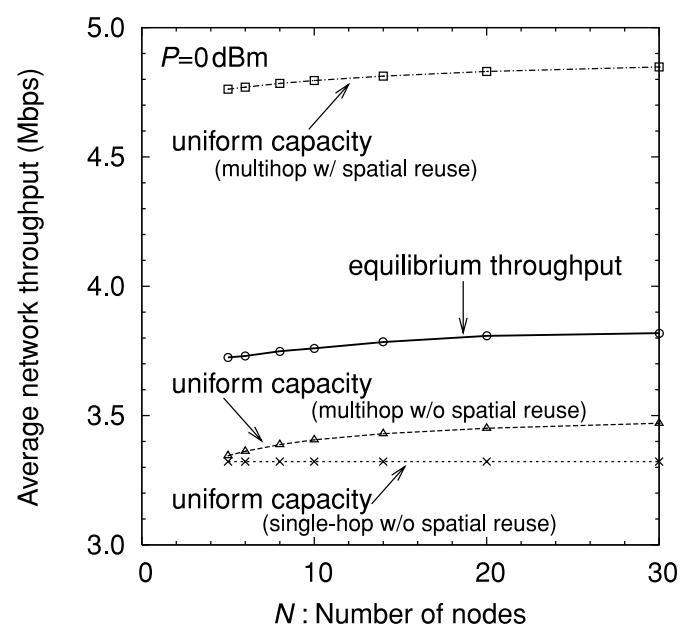

Fig. 11 Node density dependence of average network throughput.

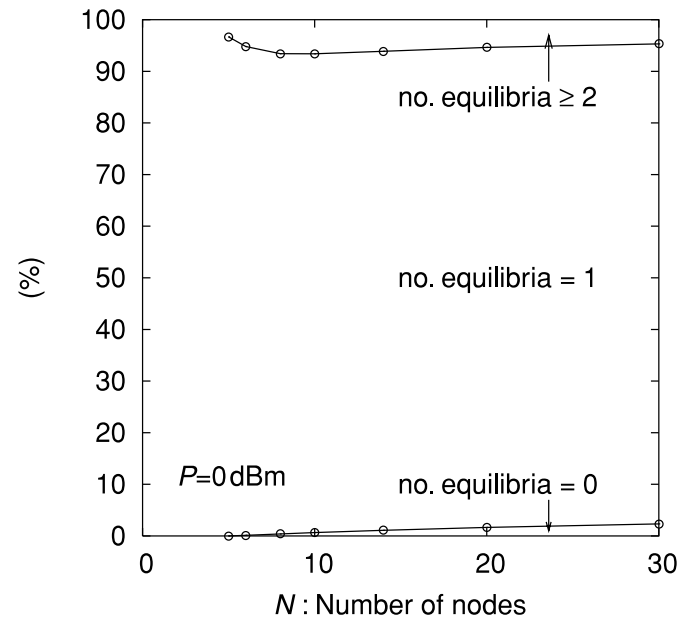

Fig. 12 Percentage of three categories based on number of equilibria as function of number of nodes.

rium throughput. If the DARS game has multiple equilibria, we use the average throughput of multiple route profiles under equilibrium conditions. With low transmit power, the DARS game attains the average throughput near the uniform capacity. With high transmit power, the equilibrium throughput is saturated because in the DARS game each source node tends to use spatial reuse, which is not effective under high power conditions.

Figure 11 shows the node density dependence of the average network throughput. The equilibrium throughput as well as the uniform capacity increases with the number of nodes. However, at the same time, a large number of nodes can lead to instability of adaptive control, as shown in Fig. 12, which shows the percentage of each category as a function of the number of nodes under the condition that all nodes transmit at the same power level $(P=0 \mathrm{dBm})$.

\section{Concluding Remarks}

We have addressed the problem of decentralized adaptive 
route selection in wireless ad hoc networks. First, we considered the end-to-end throughput of individual multihop transmission. The introduction of multihop transmission may increase per hop SNR, so that the transmission rate can be increased through the use of rate adaptation. However, the percentage of time one node can transmit a signal is reduced. Therefore, we evaluated the end-to-end throughput of multihop transmission with equally spaced nodes on a straight line. Numerical results suggest that the optimal number of hops in terms of the achievable end-toend throughput depends on the received SNR, and multihop transmission may be effective under relatively low SNR conditions.

Next, we investigated the decentralized adaptive route selection problem in which each source node wants to maximize its own throughput at the same time. We defined this problem as a DARS game. Numerical results show that the existence of Nash equilibria is not guaranteed in some cases even under the assumption that nodes have perfect information except for the knowledge of the other nodes' actual route selections. They also show that the percentage of networks in which there are no pure strategy Nash equilibria depends on the transmit power per node as well as on the spatial arrangement of the nodes.

We also evaluated network throughput under equilibrium conditions as the achievable throughput of the DARS game. Numerical results show that the DARS game attains average throughput near capacity under centralized scheduling when transmit power is low. This is because each node in the DARS game actively reuses the same bandwidth at spatially separated locations.

We would like to emphasize that the purpose of this paper was to present a means to evaluate decentralized and adaptive route selections. Further research is required to develop stable and highly efficient adaptive route selection algorithms for wireless ad hoc networks.

\section{Acknowledgments}

This work is supported in part by a Grant-in-Aid for the 21st Century COE Program (no. 14213201) of MEXT, Japan and by a Grant-in-Aid for Scientific Research (A) (no. 16206040) of JSPS, and JSPS Research Fellowships for Young Scientists.

\section{References}

[1] J. Weatherall and A. Jones, "Ubiquitous networks and their applications," IEEE Wireless Commun. Mag., vol.9, no.1, pp.18-29, Feb. 2002.

[2] R. Ramanathan and J. Redi, "A brief overview of ad hoc networks: Challenges and directions," IEEE Commun. Mag., vol.40, no.5, pp.20-22, May 2002.

[3] A.J. Goldsmith and S.B. Wicker, "Design challenges for energyconstrained ad hoc wireless networks," IEEE Wireless Commun. Mag., vol.9, no.4, pp.8-27, Aug. 2002.

[4] S. Ramanathan and M. Steenstrup, "A survey of routing techniques for mobile communications networks," ACM/Baltzer Mobile Networks and Applications, vol.1, no.2, pp.89-104, 1996.
[5] J. Broch, D.A. Maltz, D.B. Johnson, Y.C. Hu, and J. Jetcheva, "A performance comparison of multi-hop wireless ad hoc network routing protocols," Proc. Fourth Annual ACM/IEEE International Conf. on Mobile Computing and Networking (MOBICOM'98), pp.85-97, Dallas, TX, Oct. 1998.

[6] E.M. Royer and C.K. Toh, "A review of current routing protocols for ad hoc mobile wireless networks," IEEE Pers. Commun. Mag., vol.6, no.2, pp.46-55, April 1999.

[7] S. Toumpis and A.J. Goldsmith, "Performance, optimization, and cross-layer design of media access protocols for wireless ad hoc networks," Proc. IEEE ICC'03, pp.2234-2240, May 2003.

[8] S. Shakkottai, T.S. Rappaport, and P.C. Karlsson, "Cross-layer design for wireless networks," IEEE Commun. Mag., vol.41, no.10, pp.74-80, Oct. 2003.

[9] P. Gupta and P.R. Kumar, "The capacity of wireless networks," IEEE Trans. Inf. Theory, vol.46, no.2, pp.388-404, March 2000.

[10] M. Grossglauser and D.N.C. Tse, "Mobility increases the capacity of ad hoc wireless networks," IEEE/ACM Trans. Netw., vol.10, no.4, pp.477-486, Aug. 2002.

[11] S. Toumpis and A.J. Goldsmith, "Capacity regions for wireless ad hoc networks," IEEE Trans. Wireless Commun., vol.2, no.4, pp.736-748, July 2003.

[12] B. Radunovic and J.Y.L. Boudec, "Optimal power control, scheduling and routing in UWB networks," IEEE J. Sel. Areas Commun., vol.22, no.7, pp.1252-1270, Sept. 2004

[13] A.B. MacKenzie and S.B. Wicker, "Selfish users in Aloha: A gametheoretic approach," Proc. IEEE VTC 2001-Fall, pp.1354-1357, Oct. 2001.

[14] A.B. MacKenzie and S.B. Wicker, "Stability of multipacket slotted Aloha with selfish users and perfect information," Proc. IEEE Infocom'03, April 2003.

[15] V. Srinivasan, P. Nuggehalli, C.F. Chiasserini, and R.R. Rao, "Cooperation in wireless ad hoc networks," Proc. IEEE Infocom'03, no.1, pp.808-817, March 2003.

[16] S. Eidenbenz, V.A. Kumar, and S. Zust, "Equilibria in topology control games for ad hoc networks," Proc. DialM-POMC'03, pp.2-11, Sept. 2003.

[17] J. Jun, P. Peddabachagari, and M. Sichitiu, "Theoretical maximum throughput of IEEE 802.11 and its applications," Proc. 2nd IEEE International Symposium on Network Computing and Applications, pp.249-256, April 2003.

[18] J. Jun and M.L. Sichitiu, "The nominal capacity of wireless mesh networks," IEEE Wireless Commun. Mag., vol.10, no.5, pp.8-14, Oct. 2003.

[19] E. Anshelevich, A. Dasgupta, E. Tardos, and T. Wexler, "Nearoptimal network design with selfish agents," Proc. Thirty-Fifth Annual ACM Symposium on Theory of Computing, pp.511-520, 2003.

[20] D. Fudenberg and J. Tirole, Game Theory, MIT Press, 1991 


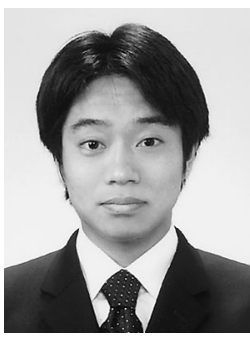

Koji Yamamoto was born in Kochi, Japan, on May 2, 1979. He received a B.E. degree in electrical and electronic engineering and an M.E. degree in informatics from Kyoto University in 2002 and 2004, respectively. He is currently a research fellow of the Japan Society for the Promotion of Science (JSPS), studying toward his Ph.D. degree at the Graduate School of Informatics, Kyoto University. His research interests include next-generation wireless communication systems and wireless ad hoc networks.

He received the IEEE VTS Japan 2003 Young Researcher's Encouragement Award and the PIMRC 2004 Best Student Paper Award. He is a student member of the IEEE.

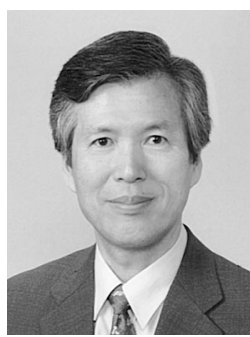

Susumu Yoshida received the B.E., M.E. and $\mathrm{Ph} . \mathrm{D}$. degrees in electrical engineering from Kyoto University, Kyoto, Japan in 1971, 1973 and 1978, respectively. Since 1973, he has been with the Faculty of Engineering, Kyoto University and currently he is a full professor of the Graduate School of Informatics, Kyoto University. During the last 20 years, he has been mainly engaged in the research of wireless personal communications. His current research interest includes wireless transmission technologies beyond IMT-2000 and wireless ad hoc networks. During 1990-1991, he was a visiting scholar at WINLAB, Rutgers University, U.S.A. and Carleton University in Canada. He served as an Executive Committee Chairperson of PIMRC'99, Osaka and also as a Technical Program Committee Chairperson of IEEE VTC 2000-Spring, Tokyo. He was a guest editor of IEEE J-SAC on Wireless Local Communications published in April and May 1996. He was a Director, Journal and Transactions of IEICE during 2002-2004. He was awarded the Young Researcher's Award in 1978 and the Achievement Award in 1993 both from the IEICE. 